\title{
Upregulation of TGF-b-induced HSP27 by HSP90 inhibitors in osteoblasts
}

Gen Kuroyanagi ( $\nabla$ kokuryugen@yahoo.co.jp )

Nagoya City University Graduate School of Medical Sciences

Haruhiko Tokuda

National Center for Geriatrics and Gerontology

Kazuhiko Fujita

Nagoya City University Graduate School of Medical Sciences

Tetsu Kawabata

Nagoya City University Graduate School of Medical Sciences

Go Sakai

Nagoya City University Graduate School of Medical Sciences

Woo Kim

Gifu University Graduate School of Medicine

Tomoyuki Hioki

Gifu University Graduate School of Medicine

Junko Tachi

Gifu University Graduate School of Medicine

Rie Matsushima-Nishiwaki

Gifu University Graduate School of Medicine

Takanobu Otsuka

Nagoya City University Graduate School of Medical Sciences

Hiroki lida

Gifu University Graduate School of Medicine

Osamu Kozawa

Gifu University Graduate School of Medicine

\section{Research Article}

Keywords: Heat shock protein, HSP90, HSP90 inhibitor, HSP27, TGF- $\beta$, SAPK/JNK, Osteoblast

Posted Date: October 19th, 2021

DOI: https://doi.org/10.21203/rs.3.rs-986636/v1 
License: (c) (i) This work is licensed under a Creative Commons Attribution 4.0 International License. Read Full License 


\section{Abstract}

Background: Heat shock protein (HSP) 90 functions as a molecular chaperone and is constitutively expressed and induced in response to stress in many cell types. We have previously demonstrated that transforming growth factor-b (TGF-b), the most abundant cytokine in bone cells, induces the expression of HSP27 through Smad2, p44/p42 mitogen-activated protein kinase (MAPK), p38 MAPK, and stressactivated protein kinase/c-Jun N-terminal kinase (SAPK/JNK) in mouse osteoblastic MC3T3-E1 cells. This study investigated the effects of HSP90 on the TGF-b-induced HSP27 expression and the underlying mechanism in mouse osteoblastic MC3T3-E1 cells.

Methods: Clonal osteoblastic MC3T3-E1 cells were treated with the HSP90 inhibitors and then stimulated with TGF-b. HSP27 expression and the phosphorylation of Smad2, p44/p42 MAPK, p38 MAPK, and SAPK/JNK were evaluated by western blot analysis.

Result: HSP90 inhibitors 17-dimethylaminoethylamino-17-demethoxy-geldanamycin (17-DMAG) and onalespib significantly enhanced the TGF-b-induced HSP27 expression. HSP90 inhibitors, geldanamycin, onalespib, and 17-DMAG did not affect the TGF-b-stimulated phosphorylation of Smad2. Geldanamycin did not affect the TGF-b-stimulated phosphorylation of p44/p42 MAPK or p38 MAPK but significantly enhanced the TGF-b-stimulated phosphorylation of SAPK/JNK. Onalespib also increased the TGF-bstimulated phosphorylation of SAPK/JNK. Furthermore, SP600125, a specific inhibitor for SAPK/JNK, significantly suppressed onalespib or geldanamycin's enhancing effect of the TGF-b-induced HSP27 expression levels. As for the canonical BMP signaling pathway, BMP-4 failed to induce the expression of HSP27 in osteoblastic MC3T3-E1 cells.

Conclusion: Our results strongly suggest that HSP90 inhibitors upregulated the TGF-b-induced HSP27 expression and that these effects of HSP90 inhibitors were mediated through SAPK/JNK pathway in osteoblasts.

\section{Introduction}

Heat shock proteins (HSPs), abundantly expressed in many cell types, are induced in response to stressful conditions such as heat stress and pathological conditions [1]. HSPs are recognized as molecular chaperones and help the folding of nascent proteins and the refolding of denatured proteins [1]. Based on the molecular sizes, HSPs are generally divided into seven major groups such as HSPH (HSP110), HSPC (HSP90), HSPA (HSP70), HSPD/E (HSP60/HSP10), CCT (TRiC), DNAJ (HSP40), and HSPB (small molecular size HSPs) [1,2]. HSP27 is a major protein in the small molecular size HSPs and works independently of ATP [1]. As an ATP-independent molecular chaperone, HSP27 binds to misfolded proteins and transfers them to the ATP-dependent chaperones, including HSP90 and HSP70 for protein refolding or to proteasomes for protein degradation $[1,3]$. Although HSP27 exists typically in the large oligomer, the conformational change to the monomer or the dimers occurs when it is phosphorylated [1]. Conversely, HSP90 is one of the most abundant proteins in human cells, comprising $1-2 \%$ of cellular 
proteins under physiological conditions and 4-6\% under stressful conditions [3,4]. HSP90 is also known as an ATP-dependent molecular chaperone and plays central roles in stabilizing and activating the client proteins [5]. HSP90, as a molecular chaperone, participates in stabilizing and functioning numerous oncogenic signaling proteins in cancer, including breast and lung cancers [5-7]. HSP90 expression is markedly increased in cancer specimens compared to the normal tissues [4,8]. Thus, inhibition of HSP90 function using an HSP90 inhibitor is now considered a therapeutic modality in treating specific cancers $[4,8]$. Using clinical trials, accumulating evidence suggests that the HSP90 inhibitors such as geldanamycin, 17-allylamino-17-demethoxy-geldanamycin (17-AAG), and 17-dimethylaminoethylamino17-demethoxy-geldanamycin (17-DMAG) can be used for the treatment of cancer disease [7]. Also, HSP90 inhibitors have been proposed as a novel class of senolytics to reduce age-related symptoms in vivo [9].

During bone remodeling, the continuous process of renewal throughout human life, bone resorption by osteoclasts is followed by bone formation by osteoblasts $[10,11]$. For retaining the volume and the strength, the process is finely balanced with coupling to ensure sufficient new bone formation at the resorption area $[10,11]$. In contrast, under pathological conditions such as osteoporosis with aging, bone resorption exceeds formation, resulting in bone loss and an increased risk of osteoporotic fractures $[10,11]$. Transforming growth factor-b (TGF-b), a member of TGF-b superfamily consists of bone morphogenic proteins and activin, is the most abundant cytokine in bone cells and plays a crucial role in bone remodeling [12]. TGF-b embedded in the bone matrix is released when osteoclasts activate bone resorption and recruit osteoblast precursors to start bone formation [12]. Regarding the signaling mechanisms, TGF-b activates Smad signaling pathways, including Smad2 and Smad3 [13], and nonSmad pathways such as p44/p42 mitogen-activated protein kinase (MAPK), p38 MAPK, and stressactivated protein kinase/c-Jun N-terminal kinase (SAPK/JNK) [14]. Our previous studies have shown that TGF-b induces the expression of HSP27 through Smad2, p44/p42 MAPK, p38 MAPK, and SAPK/JNK in mouse osteoblastic MC3T3-E1 cells [15,16].

Although HSPs functions in osteoblasts have not yet been clarified, we have already demonstrated that HSP27 down-regulates the migration of mouse osteoblastic MC3T3-E1 cells induced by PDGF-BB [17]. We have also demonstrated that HSP27 in unphosphorylated form has an inhibitory effect on osteocalcin release, while it has a stimulatory effect on mineralization in osteoblasts [18]. Regarding the HSP90 function in osteoblasts, bisphosphonates, a therapeutic tool for osteoporosis, and low-intensity pulsed ultrasound stimulation (LIPUS), a clinically used device for accelerating bone fracture healing, could reportedly induce HSP90 expression in osteoblastic cells $[19,20]$. We have already demonstrated that HSP90 inhibitors upregulate the endothelin-1-induced HSP27 expression through the SAPK/JNK pathway but not p38 MAPK in mouse osteoblastic MC3T3-E1 cells [21], and that HSP90 inhibitors enhance the prostaglandin $\mathrm{D}_{2}\left(\mathrm{PGD}_{2}\right)$-induced HSP27 expression through both the SAPK/JNK and p38 MAPK pathways in these cells [22]. However, the mechanism whereby HSP90 functions on the expression of HSP27 in osteoblasts remains unclear.

In this study, we investigated the effects of HSP90 inhibitors on the TGF-b-induced HSP27 expression and the underlying mechanism using mouse osteoblastic MC3T3-E1 cells. We identified that HSP90 inhibitors 
upregulated the TGF-b-induced HSP27 expression and that the effects were mediated through the SAPK/JNK pathway in osteoblasts.

\section{Methods}

\section{Materials}

TGF-b and BMP-4 were purchased from R\&D Systems, Inc. (Minneapolis, MN, USA). Onalespib was purchased from Selleckchem (Houston, TX, USA). Geldanamycin was obtained from Sigma-Aldrich Co. (St. Louis, MO, USA). 17-DMAG and SP600125 were purchased from Calbiochem-Novabiochem Co. (La Jolla, CA, USA). HSP27 antibodies, phosphorylated HSP27 antibodies, and glyceraldehyde-3-phosphate dehydrogenase (GAPDH) antibodies were obtained from Santa Cruz Biotechnology, Inc. (Santa Cruz, CA, USA). Phospho-specific Smad2, Smad2, phospho-specific p44/p42 MAPK, p44/p42 MAPK, phosphospecific p38 MAPK antibodies, p38 MAPK antibodies, phospho-specific SAPK/JNK antibodies, and SAPK/JNK antibodies, were obtained from Cell Signaling Technology, Inc. (Beverly, MA, USA). An ECL Western blotting detection system was obtained from GE Healthcare Life Sciences (Chalfont, UK). Other materials and chemicals were obtained from commercial sources. Onalespib, geldanamycin, 17-DMAG, and SP600125 were dissolved in dimethyl sulfoxide (DMSO). The maximum concentration of DMSO was $0.1 \%$, which did not affect the assay for Western blot analysis [21-23].

\section{Cell culture}

The osteoblastic MC3T3-E1 cells, established from neonatal mouse calvaria [24], were incubated at $37^{\circ} \mathrm{C}$ with $5 \% \mathrm{CO}_{2}$ and cultured in a-minimum essential medium (a-MEM) supplemented with $10 \%$ fetal bovine serum (FBS) as previously described [25]. Cells were seeded into 90-mm diameter dishes $\left(2 \times 10^{5}\right.$ cells/dish) in a-MEM supplemented with $10 \%$ FBS. a-MEM medium was supplemented with $0.3 \%$ FBS after five days. After 48 hours, the cells were used for the experiments. Cells of passages under 20 were selected for the experiments.

\section{Western blot analysis}

The osteoblasts were pretreated with various doses of 17-DMAG, onalespib, or geldanamycin for 60 min and then treated by 3 or $10 \mathrm{ng} / \mathrm{ml}$ of TGF-b, $30 \mathrm{ng} / \mathrm{ml}$ of BMP-4 or vehicle in a-MEM supplemented with $0.3 \%$ FBS. As previously described [21-23], the osteoblastic cells were incubated for the indicated periods and washed twice in phosphate-buffered saline (PBS). In brief, lysate containing $62.5 \mathrm{mM}$ of Tris/ $\mathrm{HCl}, \mathrm{pH}$ $6.8,2 \%$ sodium dodecyl sulfate (SDS), $50 \mathrm{mM}$ of dithiothreitol, and $10 \%$ glycerol was used to extract total protein from the cells. ${ }^{21-23}$ The cells were also homogenized and sonicated in the lysate buffer. Proteins were separated by SDS-polyacrylamide gel electrophoresis (PAGE) using Laemmli's method [26] in 10\% 
polyacrylamide gels and transferred to polyvinylidene difluoride (PVDF) membranes (Bio-Rad Laboratories, Inc., Hercules, CA, USA). Following blocking for $1 \mathrm{~h}$ at room temperature in $5 \%$ fat free dry milk in Tris-buffered saline-Tween (TBS-T; $20 \mathrm{mM}$ of Tris- $\mathrm{HCl}, \mathrm{pH} \mathrm{7.6,137} \mathrm{mM} \mathrm{of} \mathrm{NaCl}$ and $0.1 \%$ Tween 20), membranes were incubated more than $12 \mathrm{~h}$ at $4^{\circ} \mathrm{C}$ with the following primary antibodies (HSP27 antibodies (sc-1049); phospho-specific Smad2 antibodies (\#3108); Smad2 antibodies (\#3102); phosphospecific p44/p42 MAPK antibodies (\#9101); p44/p42 MAPK antibodies (\#9102); phospho-specific p38 MAPK antibodies (\#4511); p38 MAPK antibodies (\#9212); phospho-specific SAPK/JNK antibodies (\#4668); SAPK/JNK antibodies (\#9252) and GAPDH antibodies (\#60004-1-IG)). The PVDF membranes were washed three times in TBS-T. The membranes were then incubated with a secondary antibody (goat against rabbit $\lg G$ ) with $5 \%$ fat free dry milk in TBS-T for $1 \mathrm{~h}$ at room temperature and washed three times with TBS-T. Protein bands were visualized on X-ray film by ECL Western blotting detection system. As for the induction of HSP27 in osteoblast-like MC3T3-E1 cells, we have previously shown that TGF-

b significantly induces the expression of HSP27 at $12 \mathrm{~h}$ after the stimulation $[15,16]$. Conversely, regarding the intracellular signaling of TGF-b, we have also reported that TGF-b significantly induces the phosphorylation of Smad2, p44/p42 MAPK, p38 MAPK, and SAPK/JNK at $2 \mathrm{~h}$ after the stimulation [15].Therefore, in this study, we conducted the experiments about HSP27 induction in a long time point $(12 \mathrm{~h})$ and intracellular signaling in a short time point $(2 \mathrm{~h})$. The controls included the same amount of $0.1 \%$ of DMSO in this study. The vehicle for the TGF-b treatment was a solvent containing PBS, in which TGF-b was dissolved.

\section{Densitometric analysis}

Densitometric analysis for Western blotting was done by a scanner and Image J ver. 1.49 software $(\mathrm{NIH}$, Bethesda, MD, USA). Phosphorylation levels were assessed by the following method. The backgroundsubtracted signal intensity of each phosphorylation signal was normalized to the respective intensity of total protein and plotted as the fold increase compared to that in the control cells without stimulation. Regarding the quantification of HSP27 expression levels, the signal intensity of each HSP27 band was normalized to the respective intensity of GAPDH bands.

\section{Statistical analysis}

All experiments were repeated at least three times using three independent cell preparations. Three separate dishes of cells plated at three independent experiments were performed on different days. All data were presented as the mean \pm standard error of the mean (SEM) of triplicate experiment results. Differences between groups were determined by an analysis of variance followed by the Bonferroni method for multiple comparisons between pairs. $P<0.05$ was set to indicate a statistically significant difference. 


\section{Results}

HSP90 inhibitor 17-DMAG upregulates TGF-b-induced HSP27 expression in osteoblastic MC3T3-E1 cells

We have previously demonstrated that TGF-b strongly induced HSP27 expression in osteoblastic MC3T3E1 cells $[15,16]$. To investigate the effects of HSP90 on the HSP27 expression in osteoblasts, we first examined whether HSP90 inhibitor 17-DMAG [27] affects the TGF-b-induced HSP27 expression levels in mouse osteoblastic MC3T3-E1 cells. We found that treatment with $20 \mathrm{nM}$ of 17-DMAG significantly enhanced the TGF-b-induced HSP27 expression in MC3T3-E1 cells $(P=0.02)$ (Figure 1). We also found that 17-DMAG alone failed to affect the levels of HSP27 expression (Lane 1, 3, and 5).

\section{HSP90 inhibitor onalespib upregulates TGF-b-induced HSP27 expression in MC3T3-E1 cells}

Using another type of HSP90 inhibitor, onalespib [28], we further examined HSP90 effects on HSP27 expression in MC3T3-E1 cells. Similar to 17-DMAG, onalespib alone did not affect HSP27 expression levels but significantly enhanced the levels of TGF-b-induced HSP27 (20 nM: P=0.002; $30 \mathrm{nM}: \mathrm{P}=0.002)$ (Figure 2). We found that the onalespib's significant effect on HSP27 induction was observed between the 20 and $30 \mathrm{nM}$ ranges.

HSP90 inhibitors, geldanamycin, onalespib, and 17-DMAG does not affect the phosphorylation of Smad2 induced by TGF-b in osteoblastic MC3T3-E1 cells

Regarding the canonical pathways, it has been established that Smad2 and Smad3 phosphorylation is firstly required for TGF-b signal transduction [13]. Our previous study has shown that TGF-b stimulates Smad2 phosphorylation in osteoblastic MC3T3-E1 cells [29]. Thus, we next examined whether HSP90 inhibitors affect the Smad2 phosphorylation induced by TGF-b. The results showed that geldanamycin, the first HSP90 inhibitor characterized as naturally occurring [30], did not affect the Smad2 phosphorylation with ( $0.3 \mathrm{mM}: \mathrm{P}=0.93 ; 0.7 \mathrm{mM}: \mathrm{P}=0.69 ; 1.0 \mathrm{mM}: \mathrm{P}=0.68)$ or without TGFb stimulation (Figure 3A). Additionally, onalespib and 17-DMAG also did not affect Smad2 phosphorylation with (Onalespib: $\mathrm{P}=0.41$ ) or without TGF-b stimulation in MC3T3-E1 cells (Figure 3B and 3C).

Geldanamycin does not affect the phosphorylation of p44/p42 MAPK or p38 MAPK induced by TGF-b in osteoblastic MC3T3-E1 cells

In addition to the canonical pathways, it is well recognized that TGF-b can activate various other intracellular signaling pathways called non-canonical pathways, including MAPKs [14]. Our previous 
studies have shown that TGF-b induces HSP27 expression through the p44/p42 MAPK, p38 MAPK, and SAPK/JNK pathways in mouse osteoblastic MC3T3-E1 cells $[15,16]$. Therefore, we examined whether geldanamycin affects p44/p42 MAPK phosphorylation induced by TGF-b, and found that geldanamycin did not affect p44/p42 MAPK phosphorylation with (0.3 mM: $\mathrm{P}=0.82 ; 0.7 \mathrm{mM}: \mathrm{P}=0.72 ; 1.0 \mathrm{mM}: \mathrm{P}=$ 0.91) or without TGF-b stimulation in osteoblastic MC3T3-E1 cells (Figure 4A). We also examined whether geldanamycin affects p38 MAPK phosphorylation induced by TGF-b, and found that geldanamycin did not affect p38 MAPK phosphorylation with $(0.3 \mathrm{mM}: \mathrm{P}=0.15 ; 0.7 \mathrm{mM}: \mathrm{P}=0.26$; $1.0 \mathrm{mM}: \mathrm{P}=0.54$ ) or without TGF-b stimulation (Figure 4B).

HSP90 inhibitors, geldanamycin, and onalespib stimulate the phosphorylation of SAPK/JNK induced by TGF-b in osteoblastic MC3T3-E1 cells

We further examined whether HSP90 inhibitor affects SAPK/JNK phosphorylation induced by TGF-b in osteoblastic MC3T3-E1 cells. Contrary to the effects on p44/p42 MAPK and p38 MAPK, we found that geldanamycin significantly enhanced SAPK/JNK phosphorylation induced by TGF-b between 0.3 to $1.0 \mathrm{mM}(0.3 \mathrm{mM}: \mathrm{P}=0.013 ; 0.7 \mathrm{mM}: \mathrm{P}=0.004 ; 1.0 \mathrm{mM}: \mathrm{P}=0.03$ ) (Figure $5 \mathrm{~A})$. As well as geldanamycin, we also found that onalespib also significantly strengthened the phosphorylation of SAPK/JNK induced by TGF-b $(P=0.04)$ (Figure 5B).

\section{SAPK/JNK inhibitor, SP600125, suppresses the enhancing effect by onalespib and geldanamycin of the TGF-b-induced HSP27 expression in osteoblastic MC3T3-E1 cells}

To examine the involvement of SAPK/JNK in the enhancement by HSP90 inhibitor of HSP27 expression, we investigated SP600125 effects, an inhibitor for SAPK/JNK [31], on the amplification of the TGF-binduced HSP27 expression in osteoblastic MC3T3-E1 cells by onalespib. We found that SP600125 significantly inhibited the enhancement by onalespib of the TGF-b-induced HSP27 expression levels ( $P=$ 0.004) (Figure 6A). We also found that SP600125 suppressed the enhancement by geldanamycin of the TGF-b-induced HSP27 expression levels (Figure 6B).

\section{BMP-4 does not induce HSP27 expression in osteoblastic MC3T3-E1 cells}

BMP can exclusively contribute to osteogenesis in some conditions. Thus, we examined the effect of BMP-4 on HSP27 induction in osteoblast-like MC3T3-E1 cells. However, we found that BMP-4 failed to induce HSP27 expression in osteoblastic MC3T3-E1 cells $(P=0.20)$ while TGF-b significantly induced HSP27 expression $(P=0.005)$ (Figure 7).

\section{Discussion}


In the present study, HSP90's effects on the TGF-b-induced HSP27 expression were investigated using mouse osteoblastic MC3T3-E1 cells. HSP90 normally exists in many cell types, including osteoblasts [19]. We first demonstrated that HSP90 inhibition using HSP90 inhibitors such as 17-DMAG and onalespib significantly upregulated the TGF-b-induced HSP27 expression in osteoblastic MC3T3-E1 cells. As 17-DMAG and onalespib could diminish the HSP90 regulation to the TGF-b-stimulated event, it is likely that HSP90 negatively regulates the TGF-b-stimulated HSP27 induction in osteoblastic MC3T3-E1 cells.

It has been well known that TGF-b mainly activates two types of signaling pathways, such as the Smad and non-Smad pathways, also called a canonical and non-canonical pathway, respectively [13,14]. As for the Smad pathway in mouse osteoblastic MC3T3-E1 cells, our previous study showed that TGF-b actually stimulates the phosphorylation of Smad2 [29]. Thus, we examined HSP90 inhibitors' effects using geldanamycin and onalespib on Smad2 phosphorylation induced by TGF-b in MC3T3-E1 cells. We found that geldanamycin and onalespib hardly affected the TGF-b-stimulated Smad2 phosphorylation, suggesting that TGF-b-stimulated Smad2 activation is unlikely regulated by HSP90 in osteoblasts. Regarding the difference between geldanamycin and 17-DMAG, geldanamycin binds to the ATP binding site of HSP90 and subsequently prevents HSP90 activity as an HSP90 inhibitor. However, due to unacceptable hepatotoxicity, geldanamycin cannot be used in clinical practice [32]. In contrast, 17-DMAG is a semisynthetic derivative of geldanamycin and possesses reduced hepatotoxicity while retaining the molecular activities of geldanamycin [33]. We investigated the effect of 17-DMAG on the phosphorylation of Smad2 induced by TGF-b in MC3T3-E1 cells, and found that 17-DMAG did not affect the phosphorylation of Smad2 with or without TGF-b stimulation in MC3T3-E1 cells. Thus, this result also supports our hypothesis that HSP90 inhibitors do not alter the activation of Smad2 induced by TGF-b in osteoblast-like MC3T3-E1 cells.

Regarding the non-Smad pathway, we have already demonstrated that TGF-b stimulates p44/p42 MAPK, p38 MAPK, and SAPK/JNK phosphorylation in osteoblastic MC3T3-E1 cells $[15,16]$. We found that geldanamycin did not affect the TGF-b-stimulated p44/p42 MAPK or p38 MAPK phosphorylation but strongly increased the TGF-b-stimulated SAPK/JNK phosphorylation in these cells. We also confirmed that onalespib significantly enhanced SAPK/JNK phosphorylation stimulated by TGF-b. Thus, the SAPK/JNK activation is probably regulated by HSP90 in the non-canonical pathway of TGF-b in these cells. It is most likely that the upregulation by HSP90 inhibitors of the TGF-b-induced HSP27 expression is mediated by SAPK/JNK, a non-Smad pathway, in osteoblastic MC3T3-E1 cells. However, we do not have data using onalespib on p44/p42 MAPK and p38 MAPK phosphorylation induced by TGF-b. We speculated that p44/p42 MAPK and p38 MAPK might not be involved in the TGF-b-induced HSP27 in MC3T3-E1 cells based on the results treated with geldanamycin. Thus, the experiments treated with onalespib would be necessary to confirm our speculation. We have previously reported that SAPK/JNK acts as a positive regulator in HSP27 induction stimulated by TGF-b in osteoblast-like MC3T3-E1 cells $[15,16]$. Thus, the result that SP600125 markedly suppressed the TGF-b-induced HSP27 expression is consistent with our previous reports. In the present study, we showed that SP600125 significantly inhibited the enhancement by onalespib of the TGF-b-induced HSP27 expression levels. Our findings 
suggest that SP600125 truly functions as a SAPK/JNK inhibitor. Thus, it seems unlikely that SP600125 is a general HSP27 inhibitor or TGF-b-induced HSP27 expression. Our previous study showed that SAPK/JNK and p38 MAPK but not p44/p42 MAPK are involved in the upregulation by HSP90 inhibitors in the $\mathrm{PGD}_{2}$-induced HSP27 expression in these cells [22]. We have also demonstrated that SAPK/JNK but not 138 MAPK is involved in enhancing endothelin-1-induced HSP27 expression in these cells by HSP90 inhibitors. ${ }^{21}$ Therefore, as far as we know, it is likely that HSP90 regulates HSP27 expression in response to a variety of stimulations at a point upstream of SAPK/JNK commonly in mouse osteoblastic MC3T3E1 cells.

Conversely, BMP can exclusively contribute to osteogenesis in some conditions, and it is very interesting to investigate the canonical BMP signaling pathway through phospho-Smad1/5/9 when the canonical TGF-b signaling pathway through Smad2/3 is unchanged. Therefore, we examined the effect of BMP-4 on the induction of HSP27 in osteoblast-like MC3T3-E1 cells. As a result, we found that BMP-4 failed to induce HSP27 expression in these cells while TGF-b significantly induced the HSP27 expression. In our previous study, we have demonstrated that BMP-4 stimulates Smad1 phosphorylation in MC3T3-E1 cells [34]. Thus, based on our findings, it seems unlikely that the signal transduction downstream of BMP-4, including canonical BMP signaling pathway thorough phospho-Smad1/5/9, is implicated in the HSP27 induction in osteoblast-like MC3T3-E1 cells.

Although the involvement of HSP90 in bone metabolism is still unclear, bisphosphonates, a group of medicines for osteoporosis, and LIPUS, a device clinically used for non-union and fracture hearing distress, reportedly could induce the HSP90 expression in osteoblasts $[19,20]$. In contrast, it has recently been reported that HSP90 inhibition enhances bone formation and rescues glucocorticoid-induced bone loss in mice [35]. Regarding HSP27 function, we have previously shown that HSP27 in the unphosphorylated form upregulates the calcification of mouse osteoblastic MC3T3-E1 cells [18]. Taking our present findings into account, as TGF-b is known to be released from bone matrix in the process of bone resorption [12], suppression of HSP90 might likely enhance HSP27 expression induced by TGF-b in the process of bone remodeling, resulting in the upregulation of calcification essential in the osteoblastic bone formation. Our findings might provide a novel therapeutic strategy of HSP90 inhibitors to treat metabolic bone disorders, including osteoporosis, or fracture healing disturbance. Further examination will be needed to investigate the details about HSP90-effect on bone metabolism.

In summary, our results suggested that HSP90 inhibitors upregulated the TGF-b-induced HSP27 expression and that the effects of HSP90 inhibitors were mediated through the SAPK/JNK pathway in osteoblasts.

\section{Declarations}

\section{Acknowledgements}

We thank Yumiko Kurokawa for her technical assistance. 


\section{Authors' contributions}

H.T. and O.K. designed the study. G.K., K.F., T.K., T.U., G.S., W.K., T.H., J.T. and R.MN. performed experiments. G.K., T.H. and R.MN. analyzed data. G.K., H.T, and O.K. drafted the manuscript. T.O. and H.I. critically reviewed the manuscript. All authors have read and approved the final submitted manuscript.

\section{Funding}

This study was supported by Grants-in-Aid for Scientific Research (15K10487, 17K11002 and 19K18471) from the Ministry of Education, Culture, Science, Sports and Technology of Japan, and Research Funding for Longevity Sciences (28-9 and 29-12) from the National Center for Geriatrics and Gerontology, Japan.

\section{Availability of data and materials}

The datasets used and/or analyzed during the current study are available from the corresponding author on reasonable request.

\section{Ethics approval and consent to participate}

Not applicable.

\section{Consent for publication}

Not applicable.

\section{Competing interests}

The authors declare that they have no competing interests.

\section{References}

1. Kampinga HH, Hageman J, Vos MJ, Kubota H, Tanguay RM, Bruford EA, et al. Guidelines for the nomenclature of the human heat shock proteins. Cell Stress Chaperones. 2009;14:105-111. 
2. Hang K, Ye C, Chen E, Zhang W, Xue D, Pan Z. Role of the heat shock protein family in bone metabolism. Cell Stress Chaperones. 2018;23:1153-1164.

3. Sreedhar AS, Kalmar E, Csermely P, Shen YF. Hsp90 isoforms: functions, expression and clinical importance. FEBS Lett. 2004;562:11-15

4. Whitesell L, Lindquist SL. HSP90 and the chaperoning of cancer. Nat Rev Cancer. 2005;5:761-772.

5. Trepel J, Mollapour M, Giaccone G, Neckers L. Targeting the dynamic HSP90 complex in cancer. Nat Rev Cancer. 2010;10:537-549.

6. Rong B, Yang S. Molecular mechanism and targeted therapy of Hsp90 involved in lung cancer: New discoveries and developments (Review). Int J Oncol. 2018;52:321-336.

7. Haque A, Alam Q, Alam MZ, Azhar El, Sait KH, Anfinan N, et al. Current Understanding of HSP9O as a Novel Therapeutic Target: An Emerging Approach for the Treatment of Cancer. Curr Pharm Des. 2016;22:2947-2959.

8. Xu W, Neckers L. Targeting the molecular chaperone heat shock protein 90 provides a multifaceted effect on diverse cell signaling pathways of cancer cells. Clin Cancer Res. 2007;13:1625-1629.

9. Fuhrmann-Stroissnigg H, Ling YY, Zhao J, McGowan SJ, Zhu Y, Brooks RW, et al. Identification of HSP90 inhibitors as a novel class of senolytics. Nat Commun. 2017;8:422.

10. Sims NA, Morris HA, Moore RJ, Durbridge TC. Increased bone resorption precedes increased bone formation in the ovariectomized rat. Calcif Tissue Int. 1996;59:121-127.

11. Martin TJ, Sims NA. Osteoclast-derived activity in the coupling of bone formation to resorption. Trends Mol Med. 2005;11:76-81.

12. Morikawa M, Derynck R, Miyazono K. TGF-b and the TGF-b family: context-dependent roles in cell and tissue physiology. Cold Spring Harb Perspect Biol. 2016;8: pii: a021873.

13. Wu M, Chen G, Li YP. TGF-b and BMP signaling in osteoblast, skeletal development, and bone formation, homeostasis and disease. Bone Res 2016;4:16009.

14. Zhang YE. Non-Smad pathways in TGF-b signaling. Cell Res. 2009;19:128-139.

15. Hatakeyama D, Kozawa O, Niwa M, Matsuno H, Ito H, Kato K, et al. Upregulation by retinoic acid of transforming growth factor-b-stimulated heat shock protein 27 induction in osteoblasts: involvement of mitogen-activated protein kinases. Biochim Biophys Acta. 2002;1589:15-30.

16. Hayashi K, Takai S, Matsushima-Nishiwaki R, Hanai Y, Kato K, Tokuda H, et al. (-)-Epigallocatechin gallate reduces transforming growth factor b-stimulated HSP27 induction through the suppression of stress-activated protein kinase/c-Jun N-terminal kinase in osteoblasts. Life Sci. 2008;82:1012-1017. 
17. Kainuma S, Tokuda H, Yamamoto N, Kuroyanagi G, Fujita K, Kawabata T, et al. Heat shock protein 27 (HSPB1) suppresses the PDGF-BB-induced migration of osteoblasts. Int J Mol Med. 2017;40:1057-1066.

18. Kato K, Adachi S, Matsushima-Nishiwaki R, Minamitani C, Natsume H, Katagiri Y, et al. Regulation by heat shock protein 27 of osteocalcin synthesis in osteoblasts. Endocrinology. 2011;152:1872-1882.

19. Romanello M, Bivi N, Pines A, Deganuto M, Quadrifoglio F, Moro L, et al. Bisphosphonates activate nucleotide receptors signaling and induce the expression of Hsp90 in osteoblast-like cell lines. Bone. 2006;39:739-753.

20. Miyasaka M, Nakata H, Hao J, Kim YK, Kasugai S, Kuroda S. Low-intensity pulsed ultrasound stimulation enhances heat-shock protein 90 and mineralized nodule formation in mouse calvaria-derived osteoblasts. Tissue Eng Part A. 2015; 21:2829-2839.

21. Fujita K, Otsuka T, Kawabata T, Sakai G, Matsushima-Nishiwaki R, Kozawa O, et al. Inhibitors of heat shock protein 90 augment endothelin-1-induced heat shock protein 27 through the SAPK/JNK signaling pathway in osteoblasts. Mol Med Rep. 2018;17:8542-8547.

22. Kim W, Tokuda H, Kawabata T, Fujita K, Sakai G, Nakashima D, et al. Enhancement by HSP90 inhibitor of PGD2-stimulated HSP27 induction in osteoblasts: Suppression of SAPK/JNK and p38 MAP kinase. Prostaglandins Other Lipid Mediat. 2019;143:106327.

23. Fujita K, Otsuka T, Kawabata T, Kainuma S, Sakai G, Matsushima-Nishiwaki R, et al. HSP90 limits thrombin-stimulated IL-6 synthesis in osteoblast-like MC3T3-E1 cells: Regulation of p38 MAPK. Int J Mol Med. 2018;42:2185-2192.

24. Sudo H, Kodama H, Amagai Y, Yamamoto S, Kasai S. In vitro differentiation and calcification in a new clonal osteogenic cell line derived from newborn mouse calvaria. J Cell Biol. 1982;96:191-198.

25. Kozawa O, Tokuda H, Miwa M, Kotoyori J, Oiso Y. Cross-talk regulation between cyclic-AMP production and phosphoinositide hydrolysis induced by prostaglandin E2 in osteoblast-like cells. Exp Cell Res. 1992;198:130-134.

26. Laemmli UK. Cleavage of structural proteins during the assembly of the head of bacteriophage T4. Nature. 1970;227:680-685.

27. Egorin MJ, Lagattuta TF, Hamburger DR, Covey JM, White KD, Musser SM, et al. Pharmacokinetics, tissue distribution, and metabolism of 17-(dimethylaminoethylamino)-17-demethoxygeldanamycin (NSC 707545) in CD2F1 mice and Fischer 344 rats. Cancer Chemother Pharmacol. 2002;49:7-19.

28. Woodhead AJ, Angove H, Carr MG, Chessari G, Congreve M, Coyle JE, et al. Discovery of (2,4dihydroxy-5-isopropylphenyl)-[5-(4-methylpiperazin-1-ylmethyl)-1,3-dihydroisoindol-2-yl]methanone (AT13387), a novel inhibitor of the molecular chaperone Hsp90 by fragment based drug design. J Med Chem. 2010;53:5956-5969. 
29. Yamamoto N, Otsuka T, Kondo A, Matsushima-Nishiwaki R, Kuroyanagi G, Kozawa O, et al. Rac limits TGF- $\beta$-induced VEGF synthesis in osteoblasts. Mol Cell Endocrinol. 2015;405:35-41.

30. Ochel HJ, Eichhorn K, Gademann G. Geldanamycin: the prototype of a class of antitumor drugs targeting the heat shock protein 90 family of molecular chaperones. Cell Stress Chaperones. 2001;6:105112.

31. Bennett BL, Sasaki DT, Murray BW, O'Leary EC, Sakata ST, Xu W, et al. SP600125, an anthrapyrazolone inhibitor of Jun N-terminal kinase. Proc Natl Acad Sci U S A. 2001;98:13681-13686.

32. Smith V, Sausville EA, Camalier FR, Fiebig HH, Burger AM. Comparison of 17dimethylaminoethylamino-17-demethoxy-geldanamycin (17DMAG) and 17-allylamino-17demethoxygeldanamycin (17AAG) in vitro: effects on Hsp90 and client proteins in melanoma models. Cancer Chemother Pharmacol. 2005; 56:126-137.

33. Mellatyar H, Talaei S, Pilehvar-Soltanahmadi Y, Barzegar A, Akbarzadeh A, Shahabi A, et al. Targeted cancer therapy through 17-DMAG as an Hsp90 inhibitor: Overview and current state of the art. Biomed Pharmacother. 2018;102:608-617.

34. Kozawa O, Hatakeyama D, Tokuda H, Oiso Y, Matsuno H, Uematsu T. Sphingomyelinase amplifies BMP-4-induced osteocalcin synthesis in osteoblasts: role of ceramide. Cell Signal. 2002;14:999-1004.

35. Chen H, Xing J, Hu X, Chen L, Lv H, Xu C, et al. Inhibition of heat shock protein 90 rescues glucocorticoid-induced bone loss through enhancing bone formation. J Steroid Biochem Mol Biol. 2017;171:236-246.

\section{Figures}




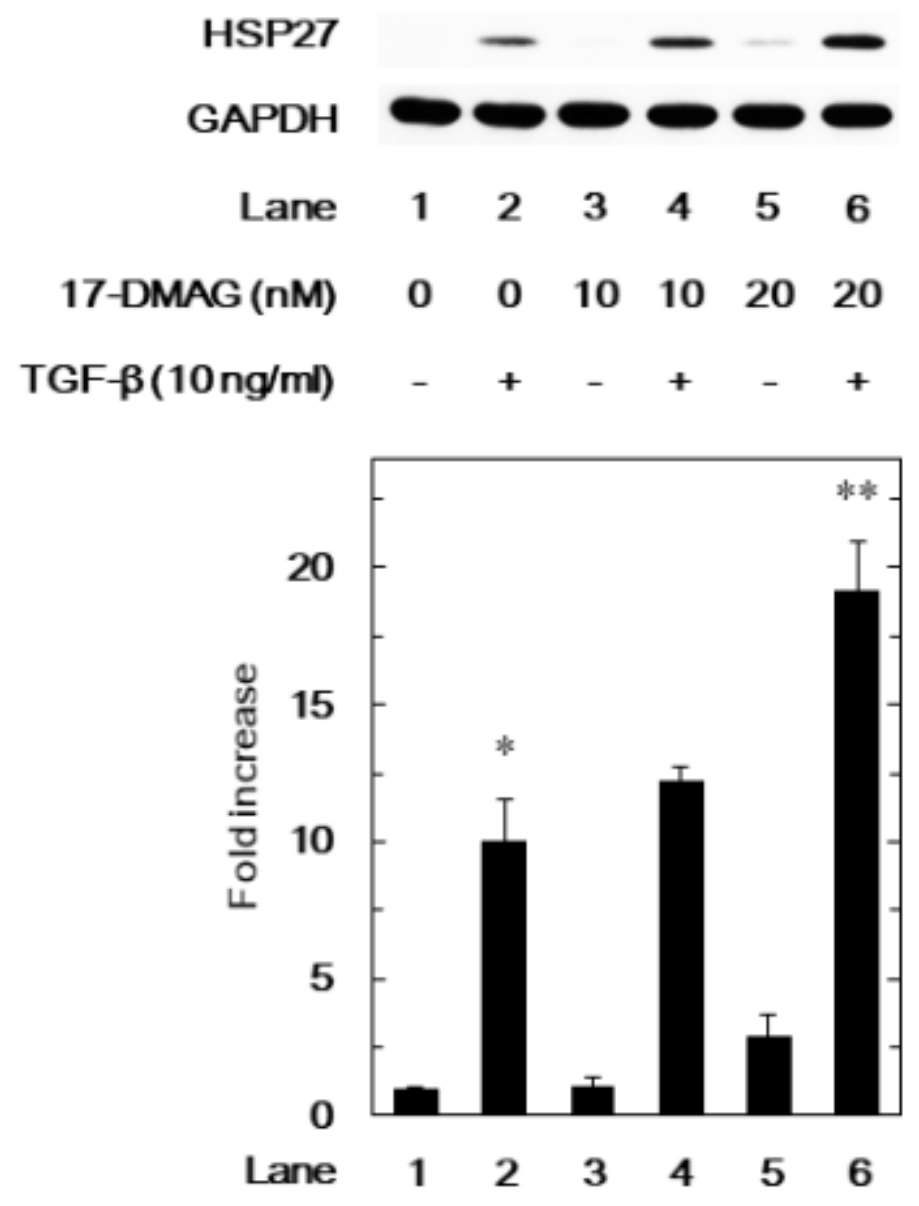

\section{Figure 1}

HSP90 inhibitor 17-DMAG upregulates TGF- $\beta$-induced HSP27 expression in osteoblastic MC3T3-E1 cells. The cultured osteoblasts were pretreated with 10 or $20 \mathrm{nM}$ of 17-DMAG for $60 \mathrm{~min}$, and subsequently incubated by $10 \mathrm{ng} / \mathrm{ml}$ of TGF- $\beta$ or vehicle for $12 \mathrm{~h}$. Cell extracts were analyzed by SDS-PAGE and Western blotting using antibodies of HSP27 or GAPDH. The histogram shows the quantitative representations of the levels of HSP27 normalized with each GAPDH gained from laser densitometric analysis. The levels were expressed as the fold increase to the basal levels presented as lane 1 . Triplicate determinations of Western blot analysis were performed corresponding to three independent cell preparations. Each value represents the mean \pm S.E.M. of triplicate determinations from three independent cell preparations. ${ }^{*} P<0.05$, compared to the value of the control cells without TGF- $\beta$ stimulation. ${ }^{* \star} \mathrm{P}<0.05$, compared to the value of TGF- $\beta$ alone. 


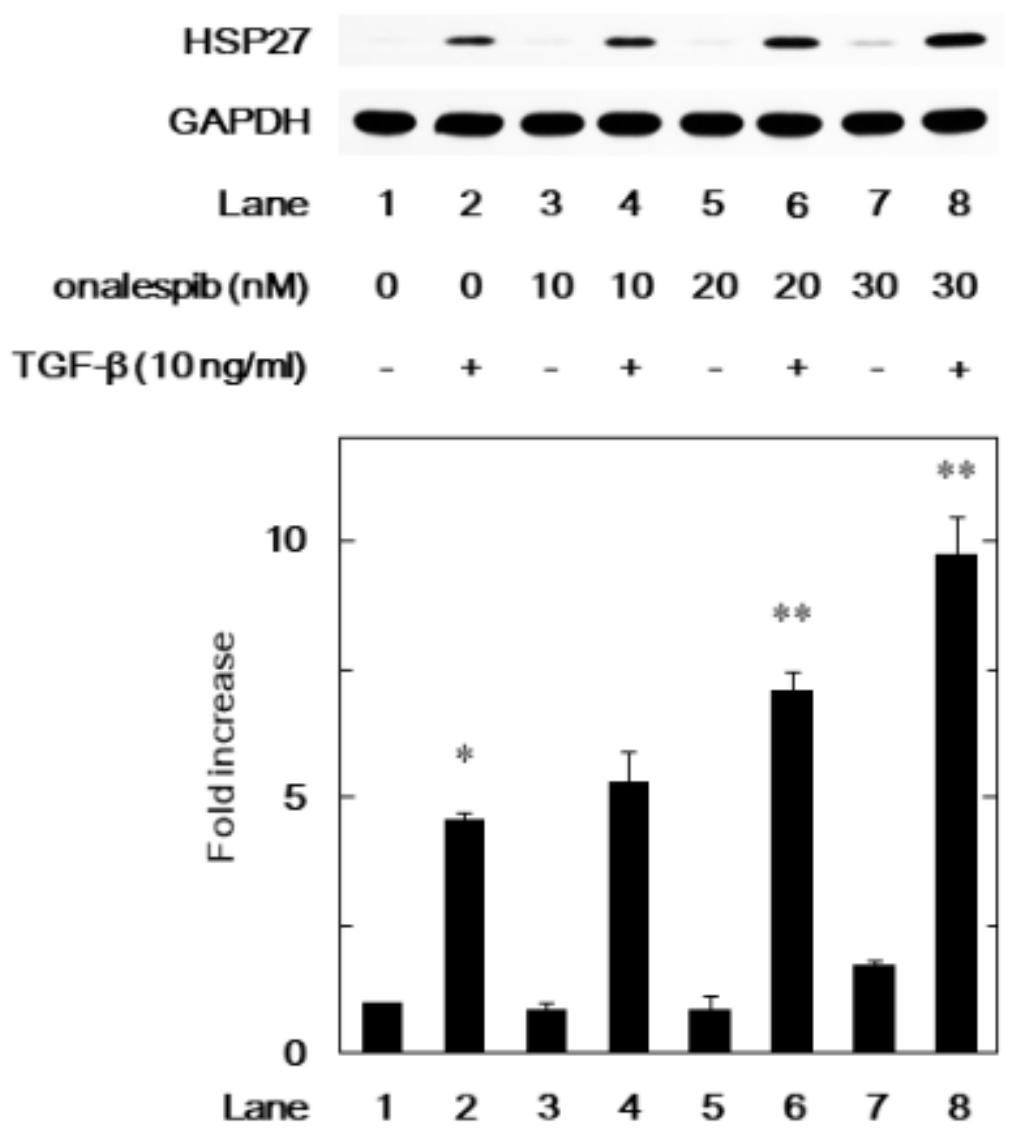

Figure 2

HSP90 inhibitor onalespib upregulates TGF- $\beta$-induced HSP27 expression in MC3T3-E1 cells. The cultured osteoblasts were pretreated with 10, 20 or $30 \mathrm{nM}$ of onalespib for $60 \mathrm{~min}$, and subsequently incubated by $10 \mathrm{ng} / \mathrm{ml}$ of TGF- $\beta$ or vehicle for $12 \mathrm{~h}$. Cell extracts were analyzed by SDS-PAGE and Western blotting using antibodies of HSP27 or GAPDH. The histogram shows the quantitative representations of the levels of HSP27 normalized with each GAPDH gained from laser densitometric analysis. The levels were expressed as the fold increase to the basal levels presented as lane 1. Triplicate determinations of Western blot analysis were performed corresponding to three independent cell preparations. Each value represents the mean \pm S.E.M. of triplicate determinations from three independent cell preparations. ${ }^{*} \mathrm{P}<$ 0.05 , compared to the value of the control cells without TGF- $\beta$-stimulation. $* \star P<0.05$, compared to the value of TGF- $\beta$ alone. 


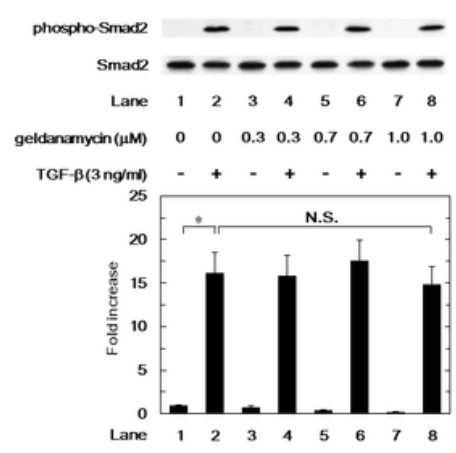

Fig.3A

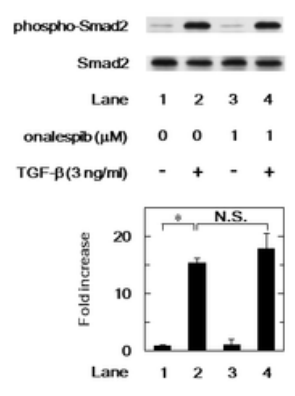

Fig.3B

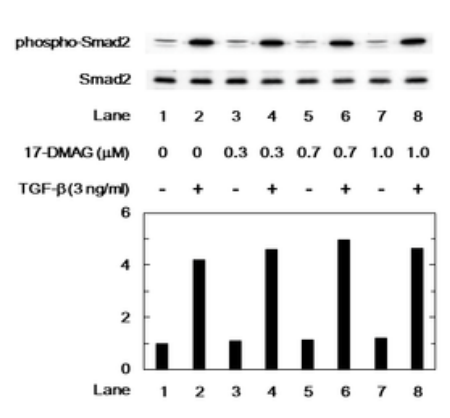

Fig.3C

\section{Figure 3}

HSP90 inhibitors, geldanamycin, onalespib and 17-DMAG does not affect the phosphorylation of Smad2 induced by TGF- $\beta$ in osteoblastic MC3T3-E1 cells. The cultured osteoblasts were pretreated with $0.3,0.7$ or $1.0 \mu \mathrm{M}$ of geldanamycin (A), $1 \mu \mathrm{M}$ of onalespib (B), $0.3,0.7$ or $1.0 \mu \mathrm{M}$ of 17-DMAG (C) or vehicle for 60 $\mathrm{min}$, and then incubated by $3 \mathrm{ng} / \mathrm{ml}$ of TGF- $\beta$ or vehicle for $60 \mathrm{~min}$. Cell extracts were analyzed by SDSPAGE and Western blotting using antibodies of phospho-specific Smad2 or Smad2. The histogram shows the quantitative representations of phosphorylated Smad2 normalized with each total Smad2 gained from laser densitometric analysis. The levels were expressed as the fold increase to the basal levels presented as lane 1. (A, B) Triplicate determinations of Western blot analysis were performed corresponding to three independent cell preparations. Each value represents the mean \pm S.E.M. of triplicate determinations from three independent cell preparations. N.S. means no significant difference between the indicated pairs. 

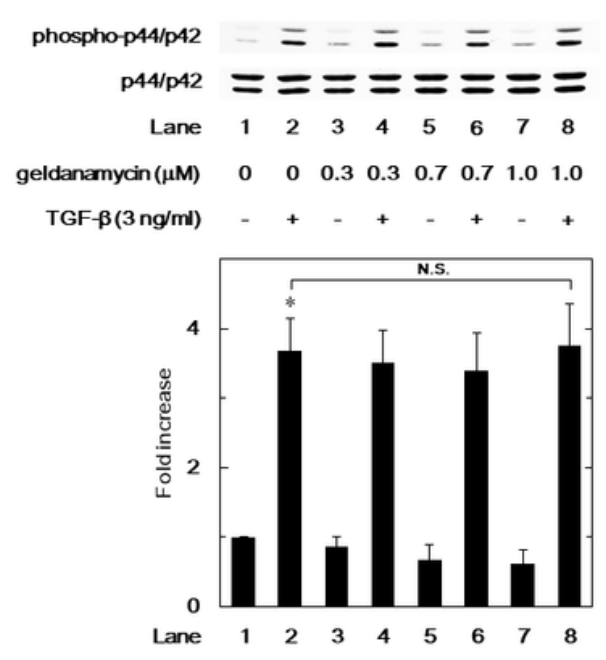

Fig.4A
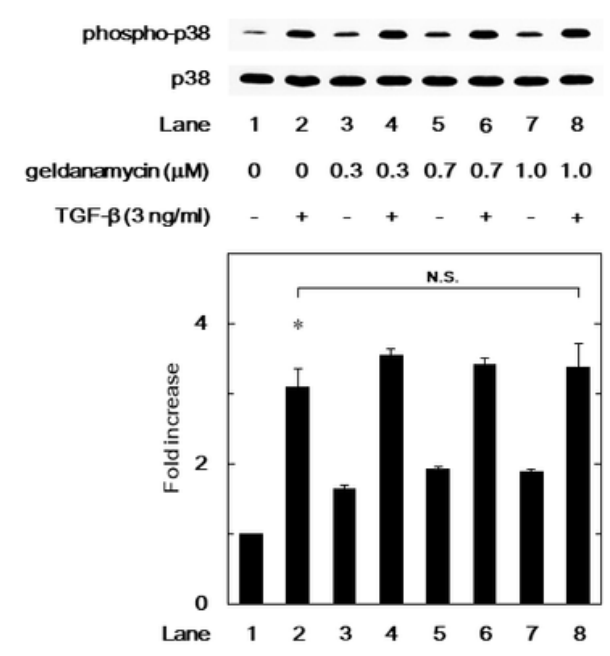

Fig.4B

\section{Figure 4}

Geldanamycin does not affect the phosphorylation of p44/p42 MAPK or p38 MAPK induced by TGF- $\beta$ in osteoblastic MC3T3-E1 cells. The cultured osteoblasts were pretreated with $0.3,0.7$ or $1.0 \mu \mathrm{M}$ of geldanamycin for $60 \mathrm{~min}$, and then incubated by $3 \mathrm{ng} / \mathrm{ml}$ of TGF- $\beta$ or vehicle for $2 \mathrm{~h}$. Cell extracts were analyzed by SDS-PAGE and Western blotting using antibodies of phospho-specific p44/p42 MAPK or p44/p42 MAPK (A) or phospho-specific p38 MAPK or p38 MAPK (B). The histogram shows the quantitative representations of phosphorylated p44/p42 MAPK normalized with each total p44/p42 MAPK (A) or the levels of phosphorylated p38 MAPK normalized with each total p38 MAPK (B) gained from laser densitometric analysis. The levels were expressed as the fold increase to the basal levels presented as lane 1. Triplicate determinations of Western blot analysis were performed corresponding to three independent cell preparations. Each value represents the mean \pm S.E.M. of triplicate determinations from three independent cell preparations. N.S. means no significant difference between the indicated pairs. 


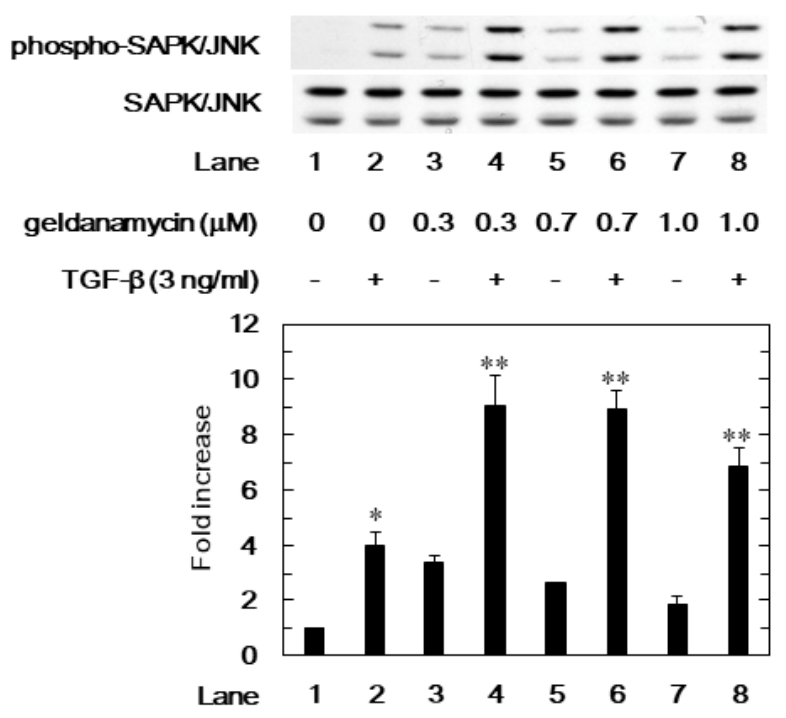

Fig. 5A
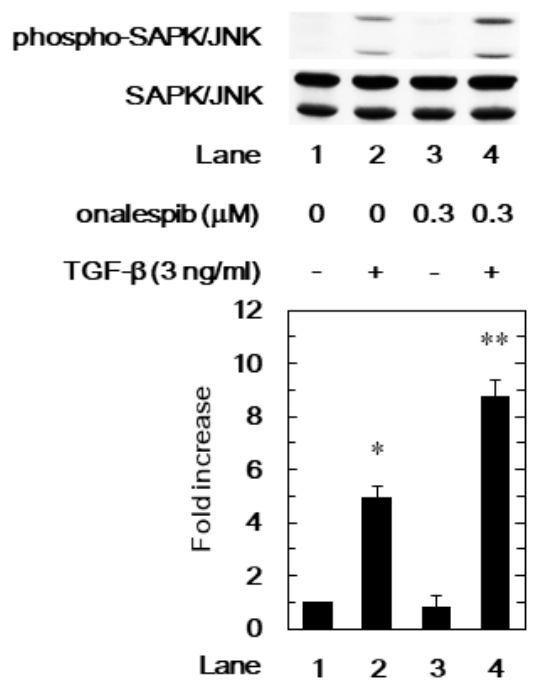

Fig.5B

Figure 5

HSP90 inhibitors, geldanamycin and onalespib, stimulate the phosphorylation of SAPK/JNK induced by TGF- $\beta$ in osteoblastic MC3T3-E1 cells. The cultured osteoblasts were pretreated with $0.3,0.7,1.0 \mu \mathrm{M}$ of geldanamycin (A) and $0.3, \mu \mathrm{M}$ of onalespib (B) for $60 \mathrm{~min}$, and then incubated by $3 \mathrm{ng} / \mathrm{ml}$ of TGF- $\beta$ or vehicle for $2 \mathrm{~h}$. Cell extracts were analyzed by SDS-PAGE and Western blotting using antibodies of phospho-specific SAPK/JNK or SAPK/JNK. The histogram shows the quantitative representations of the levels of phosphorylated SAPK/JNK normalized with each total SAPK/JNK gained from laser densitometric analysis. The levels were expressed as the fold increase to the basal levels presented as lane 1. Triplicate determinations of Western blot analysis were performed corresponding to three independent cell preparations. Each value represents the mean \pm S.E.M. of triplicate determinations from three independent cell preparations. ${ }^{*} P<0.05$, compared to the value of the control cells without TGF- $\beta$ stimulation. ${ }^{* *} \mathrm{P}<0.05$, compared to the value of TGF- $\beta$ alone. 

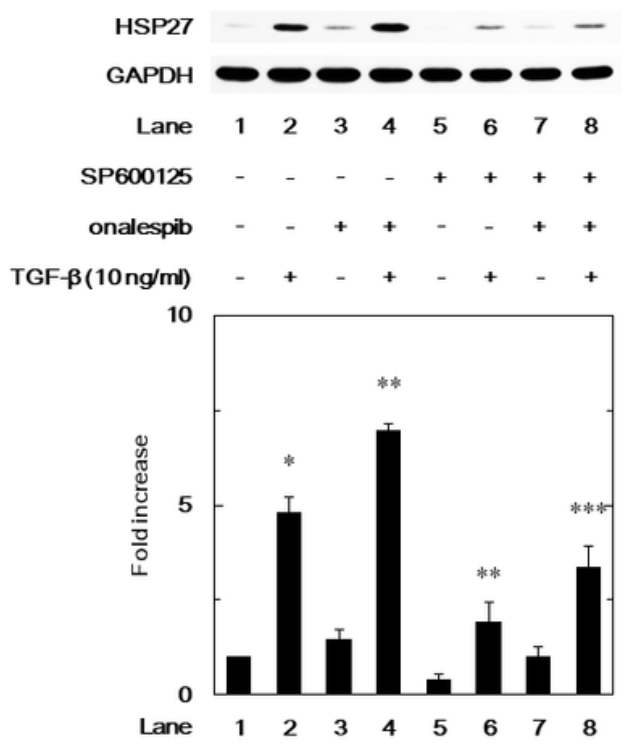

Fig.6A

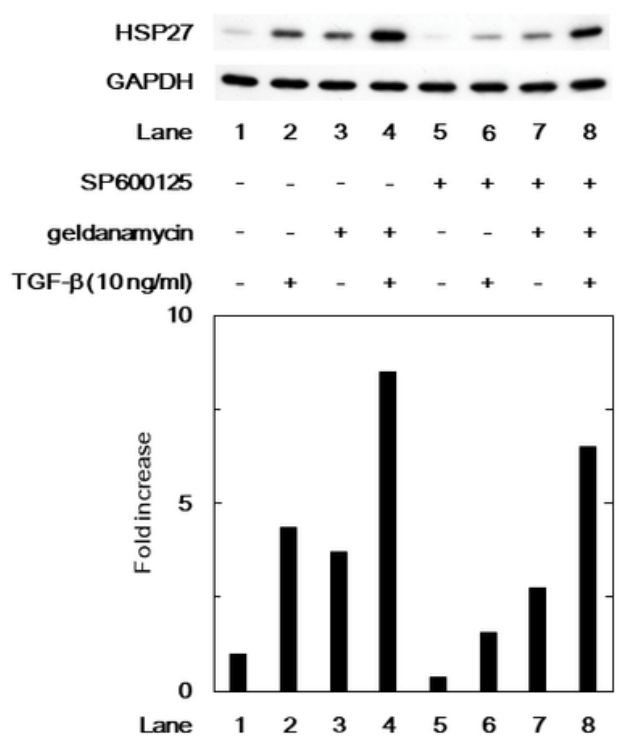

Fig.6B

\section{Figure 6}

SAPK/JNK inhibitor, SP600125, suppresses the enhancing effect by onalespib and geldanamycin of the TGF- $\beta$-induced HSP27 expression in osteoblastic MC3T3-E1 cells. The cultured osteoblasts were preincubated with $10 \mu \mathrm{M}$ of SP600125 or vehicle for $60 \mathrm{~min}$. The cells were subsequently pretreated with 30 $\mathrm{nM}$ of onalespib (A), $30 \mathrm{nM}$ of geldanamycin (B) or vehicle for $60 \mathrm{~min}$, and then stimulated by $10 \mathrm{ng} / \mathrm{ml}$ of TGF- $\beta$ or vehicle for $12 \mathrm{~h}$. Cell extracts were analyzed by SDS-PAGE and Western blotting using antibodies of HSP27 or GAPDH. The histogram shows the quantitative representations of HSP27 levels normalized with each GAPDH gained from laser densitometric analysis. The levels were expressed as the fold increase to the basal levels presented as lane 1. (A) Triplicate determinations of Western blot analysis were performed corresponding to three independent cell preparations. Each value represents the mean \pm S.E.M. of triplicate determinations from three independent cell preparations. ${ }^{*} P<0.05$, compared to the value of the control cells without TGF- $\beta$-stimulation. ${ }^{*} \mathrm{P}<0.05$, compared to the value of TGF- $\beta$ alone. ${ }^{* \star} \mathrm{P}<0.05$, compared to the value of TGF- $\beta$ and onalespib treatment. 


\section{HSP27}

\section{GAPDH}

\section{Lane $\quad 1 \quad 2 \quad 3$}

\section{stimulator - BMP-4TGF- $\beta$}

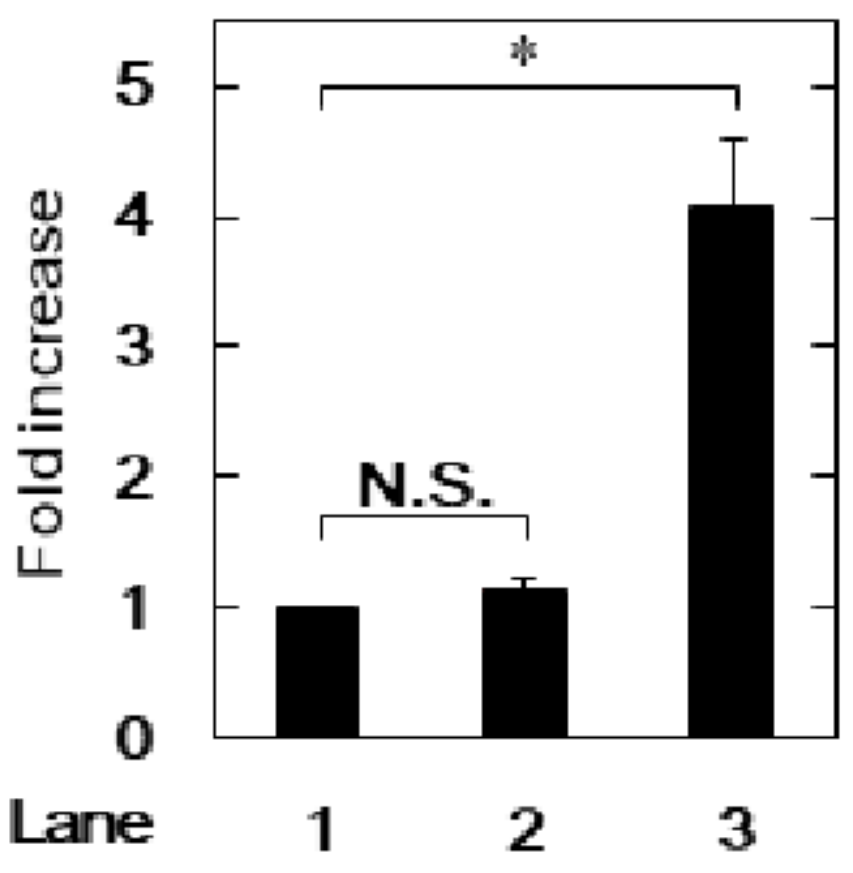

Figure 7

BMP-4 does not induce HSP27 expression in osteoblastic MC3T3-E1 cells. The cultured osteoblasts were treated by $30 \mathrm{ng} / \mathrm{ml}$ of BMP-4, $10 \mathrm{ng} / \mathrm{ml}$ of TGF- $\beta$ or vehicle for $12 \mathrm{~h}$. Cell extracts were analyzed by SDSPAGE and Western blotting using antibodies of HSP27 or GAPDH. The histogram shows the quantitative representations of the levels of HSP27 normalized with each GAPDH gained from laser densitometric analysis. The levels were expressed as the fold increase to the basal levels presented as lane 1. Triplicate determinations of Western blot analysis were performed corresponding to three independent cell preparations. Each value represents the mean \pm S.E.M. of triplicate determinations from three independent cell preparations. ${ }^{*} P<0.05$, compared to the value of the control cells without TGF- $\beta$ stimulation. N.S. means no significant difference between the indicated pairs. 